\title{
On the transverse diffusion of beams of photons in radiation therapy
}

\author{
T. Pichard, B. Dubroca, S. Brull, and M. Frank
}

\begin{abstract}
Typical external radiotherapy treatments consist in emitting beams of energetic photons targeting the tumor cells. Those photons are transported through the medium and interact with it. Such interactions affect the motion of the photons but they are typically weakly deflected which is not well modeled by standard numerical methods.

The present work deals with the transport of photons in water. The motion of those particles is modeled by an entropy-based moment model, i.e. the $M_{1}$ model. The main difficulty when constructing numerical approaches for photon beam modelling emerges from the significant difference of magnitude between the diffusion effects in the forward and transverse directions. A numerical method for the $M_{1}$ equations is proposed with a special focus on the numerical diffusion effects.
\end{abstract}

\section{Introduction}

Radiotherapy treatments consist in emitting radiations to target cancer cells. Such radiations deposit energy in the medium, so-called dose, which is responsible for biological effects (see e.g. [18]). Radiations can be seen as beams of energetic particles traveling through a medium. Here, the motion of photons modeled by a linear Boltzmann equation is focused on. Solving directly such kinetic equations requires

T. Pichard

LJLL, Université Pierre et Marie Curie, 4 Place Jussieu, 75004, Paris, France, e-mail: pichard@ann.jussieu.fr

B. Dubroca

CELIA, Université de Bordeaux, 351 cours de la libération, 33400, Talence, France

S. Brull

IMB, Université de Bordeaux, 351 cours de la libération, 33400, Talence, France

M. Frank

MathCCES, RWTH Aachen University, Schinkelstr. 2, 52062, Aachen, Germany 
high computational powers. As an alternative, the method of moments is used, leading to the so-called $M_{1}$ model. At the numerical level, such a model is cheaper than kinetic ones. However moment equations require particular considerations because they are nonlinear and their solution is constrained by a realizability condition (specified below).

The present work is a follow-up to $[5,16,15]$ which is devoted to adapt the numerical scheme presented in [15] to accurately model beams of photons. Such beams travel almost straight in a human-sized medium. The main difficulty emerges from the difference of magnitude of the diffusion effects in the forward direction and in the direction normal to the beam. Standard numerical methods typically overestimate the transverse diffusion which affects the accuracy of the results.

In the next section, the motion of photons is modeled, through kinetic and $M_{1}$ models. A standard numerical method is presented in section 3, and tested in Section 4. The problem of the transverse diffusion is presented and solved in Section 5. The last section is devoted to conclusion.

\section{Photon transport models}

For the sake of simplicity, only the motion of the photon is studied. The photons are assumed to collide only with atoms of the background medium.

\subsection{A kinetic model}

At the kinetic level, the motion of the photons is modeled by the fluence $\psi$ of photons, which satisfies the following linear Boltzmann equation

$\Omega . \nabla_{x} \psi(\varepsilon, x, \Omega)=\int_{\varepsilon}^{\varepsilon_{\max }} \int_{S^{2}} \sigma\left(\varepsilon^{\prime}, \varepsilon, \Omega^{\prime} . \Omega\right) \psi\left(\varepsilon^{\prime}, x, \Omega^{\prime}\right) d \Omega^{\prime} d \varepsilon^{\prime}-\sigma_{T}(\varepsilon) \psi(\varepsilon, x, \Omega)$,

where $\psi$ depends on energy $\varepsilon \in\left[\varepsilon_{\min }, \varepsilon_{\max }\right]$, position $x \in Z \subset \mathbb{R}^{3}$ and direction of flight $\Omega \in S^{2}$. The physical parameters $\sigma$ and $\sigma_{T}$ are called respectively differential and total cross sections, and they are chosen to model Compton collisions ([4]) as this effect is predominant in the considered energy range. Other effects may be considered for further applications.

In this equation, the $\varepsilon$ variable is considered similarily as a numerical time, and due to the energy integral in (1), such equation is solved backward in energy, from a maximum energy $\varepsilon_{\max }$ to a minimum one $\varepsilon_{\min }$.

Discretizing directly this equation is computationally too expensive for application in medical centers. For this purpose, the method of moments is applied. 


\subsection{The $M_{1}$ model}

The method of moments consists in studying angular moments, i.e. weighted integrals of $\psi$ according to the variable $\Omega$, instead of the fluence itself. Those moments depends on less variables, and are therefore typically cheaper at the computational level. The moments of $\psi$ of order up to two are defined by

$$
\psi^{0}=\int_{S^{2}} \psi(\Omega) d \Omega, \quad \psi^{1}=\int_{S^{2}} \Omega \psi(\Omega) d \Omega, \quad \psi^{2}=\int_{S^{2}} \Omega \otimes \Omega \psi(\Omega) d \Omega .
$$

According to (1), the moments of $\psi$ satisfies the following equations

$$
\begin{gathered}
\nabla_{x} \cdot \psi^{1}(\varepsilon, x)=\int_{\varepsilon}^{\varepsilon_{\max }} \sigma^{0}\left(\varepsilon^{\prime}, \varepsilon\right) \psi^{0}\left(\varepsilon^{\prime}, x\right) d \varepsilon^{\prime}-\sigma_{T}(\varepsilon) \psi^{0}(\varepsilon, x) \\
\nabla_{x} \cdot \psi^{2}(\varepsilon, x)=\int_{\varepsilon}^{\varepsilon_{\max }} \sigma^{1}\left(\varepsilon^{\prime}, \varepsilon\right) \psi^{1}\left(\varepsilon^{\prime}, x\right) d \varepsilon^{\prime}-\sigma_{T}(\varepsilon) \psi^{1}(\varepsilon, x), \\
\sigma^{0}\left(\varepsilon^{\prime}, \varepsilon\right)=2 \pi \int_{-1}^{+1} \sigma\left(\varepsilon^{\prime}, \varepsilon, \mu\right) d \mu, \quad \sigma^{1}\left(\varepsilon^{\prime}, \varepsilon\right)=2 \pi \int_{-1}^{+1} \mu \sigma\left(\varepsilon^{\prime}, \varepsilon, \mu\right) d \mu .
\end{gathered}
$$

The system (3) has more unknowns than equations. In order to solve such an undertermined system, one typically closes it by expressing the moment $\psi^{2}$ as a function of $\psi^{0}$ and $\psi^{1}$. For the present application, the entropy-based closure ([13]) was prefered as it provides desirable properties (hyperbolicity, entropy decay, correct modelling of beams). This closure, leading to the so-called $M_{1}$ closure, consists in defining $\psi^{2}$ as the second order moment of the ansatz $\psi_{M_{1}}$ minimizing Boltzmann entropy under the following constraints

$$
\begin{aligned}
\psi^{2} & =\int_{S^{2}} \Omega \otimes \Omega \psi_{M_{1}}(\Omega) d \Omega, \\
\psi_{M_{1}} & =\underset{f \in \mathscr{C}\left(\psi^{0}, \psi^{1}\right)}{\operatorname{argmin}} f \log f-f, \\
\mathscr{C}\left(\psi^{0}, \psi^{1}\right) & =\left\{f \in L^{1}\left(S^{2}\right), \quad f \geq 0, \quad \int_{S^{2}} f(\Omega) d \Omega, \quad \int_{S^{2}} \Omega f(\Omega) d \Omega\right\} .
\end{aligned}
$$

The ansatz $\psi_{M_{1}}$ can be proved to have the form $([3,12,9,17])$

$$
\psi_{M_{1}}=\exp (\Lambda \cdot \mathbf{m}(\Omega))
$$

where $\mathbf{m}(\Omega)=\left(1, \Omega_{1}, \Omega_{2}, \Omega_{3}\right)^{T}$ and $\Lambda \in \mathbb{R}^{4}$. However, the minimization problem (5) has a solution if and only if the set $\mathscr{C}\left(\psi^{0}, \psi^{1}\right)$ is non-empty.

Proposition 1 ([10]). The problem (5) has a solution if and only if the moments $\left(\psi^{0}, \psi^{1}\right)$ are in the realizability domain $\mathscr{R}_{\mathbf{m}}$ caracterized by

$$
\mathscr{R}_{\mathbf{m}}=\left\{\left(\psi^{0}, \psi^{1}\right) \in \mathbb{R} \times \mathbb{R}^{3}, \quad \text { s.t. } \quad \psi^{0}>\left|\psi^{1}\right|\right\} .
$$

For writing purposes, one rewrites (3) under the form 


$$
\begin{aligned}
& \nabla_{x} . F(\Psi)(\varepsilon, x)=\int_{\varepsilon}^{\varepsilon_{\max }} \Sigma(\varepsilon) \Psi(\varepsilon, x) d \varepsilon-\sigma_{T}(\varepsilon) \Psi(\varepsilon, x), \\
& \Psi=\left(\psi^{0}, \psi^{1}\right)^{T} \equiv \int_{S^{2}} \mathbf{m}(\Omega) \psi_{M_{1}}(\Omega) d \Omega, \quad \Sigma=\left(\begin{array}{cc}
\sigma^{0} & 0_{\mathbb{R}^{3}}^{T} \\
0_{\mathbb{R}^{3}} & \sigma^{1} I d
\end{array}\right), \\
& F=\left(\psi^{1}, \psi^{2}\right)^{T} \equiv \int_{S^{2}} \Omega \otimes \mathbf{m}(\Omega) \psi_{M_{1}}(\Omega) d \Omega .
\end{aligned}
$$

Writing the ansatz $\psi_{M_{1}}$ under the form (6), one proves that the fluxes $F$ are those of a symmetric hyberbolic equation $([12,7])$.

\section{Numerical approach}

In order to handle the non-linearity in (8), the relaxation approach proposed in [16] and based on the previous work of $[14,1]$ is used.

\subsection{Relaxation method}

The relaxation approximation leads to studying linear equations instead of (8). Let us chose $J$ directions of relaxation $\lambda_{i} \in \mathbb{R}^{N}$ and equilibrium functions $\mathbf{M}_{i}(\Psi)$ commonly called Maxwellians. With those relaxation parameters, define the relaxed equations for (8)

$$
\lambda_{i} \cdot \nabla_{x} \mathbf{f}_{i}^{\tau}(\varepsilon, x)-\left(\int_{\varepsilon}^{\varepsilon_{\max }} \Sigma(\varepsilon) \mathbf{f}_{i}^{\tau}(\varepsilon, x) d \varepsilon-\sigma_{T}(\varepsilon) \mathbf{f}_{i}^{\tau}(\varepsilon, x)\right)=\frac{\mathbf{M}_{i}\left(\sum_{i=1}^{J} \mathbf{f}_{i}^{\tau}\right)-\mathbf{f}_{i}^{\tau}}{\tau}
$$

In $[14,1]$, the authors showed for similar equations that

$$
\lim _{\tau \rightarrow 0} \sum_{i=1}^{J} \mathbf{f}_{i}^{\tau}=\Psi
$$

where the $\mathbf{f}_{j}^{\tau}$ solve (9), and under the conditions

$$
\begin{array}{r}
\forall n \in S^{2}, \quad S p\left(\partial_{\Psi} \mathbf{F}_{n}(\Psi)\right) \subset\left[\min _{i=1, \ldots, J} \lambda_{j} \cdot n, \max _{i=1, \ldots, J} \lambda_{j} . n\right] \\
\sum_{i=1}^{J} \mathbf{M}_{i}(\Psi)=\Psi, \quad \sum_{i=1}^{J} \lambda_{i} \otimes \mathbf{M}_{i}(\Psi)=F(\Psi),
\end{array}
$$

where $\mathbf{F}_{n}=\left(\psi^{1} . n, \psi^{2} n\right)$. For the present applications, we also require that the Maxwellians $\mathbf{M}_{i}: \mathscr{R}_{\mathbf{m}} \rightarrow \mathscr{R}_{\mathbf{m}}$ are realizable. 
As a first approach, one may use the following two propositions to define relaxation parameters.

Proposition 2. [2] The eigenvalues of the Jacobian of the fluxes are bounded by 1

$$
\forall n \in S^{2}, \quad S p\left(\partial_{\Psi} \mathbf{F}_{n}(\Psi)\right) \subset[-1,1] .
$$

Proposition 3. [16] The following vector is realizable

$$
\forall n \in S^{2}, \quad \forall \Psi \in \mathscr{R}_{\mathbf{m}}, \quad \Psi+\mathbf{F}_{n}(\Psi) \in \mathscr{R}_{\mathbf{m}}
$$

As a first approach, we propose to chose $2 \mathrm{~N}$ directions ( $\mathrm{N}$ being the number of space dimensions) of relaxations and to define

$$
\lambda_{i}=N e_{i}, \quad \lambda_{i+N}=-N e_{i}, \quad \mathbf{M}_{i}=\frac{\Psi+\mathbf{F}_{e_{i}}(\Psi)}{N}, \quad \mathbf{M}_{i+N}=\frac{\Psi-\mathbf{F}_{e_{i}}(\Psi)}{N} .
$$

One verifies using the last two propositions that those parameters verify (10).

\subsection{A numerical scheme for $2 D$ equations}

In the following, we focus on a $2 \mathrm{D}$ problem $(N=2)$ and the notations are adapted to $2 \mathrm{D}$ equations. However the method can straighforwardly be extended to $3 \mathrm{D}$ problems. The superscript $n$ refers to the energy step $\varepsilon^{n}$, the subscripts $l$ and $m$ refer to the position $x_{l, m}$ respectively according to the first and second cartesian axis $e_{1}$ and $e_{2}$. A numerical scheme for (8) is obtained using a splitting method on (9).

1. At the entry $\varepsilon^{n}$ of each energy cell, the values of $\mathbf{f}_{i}^{n}$ are initialized at the values of the associated Maxwellians $\mathbf{M}_{i}\left(\Psi^{n}\right)$.

2. Then one solves the homogeneous relaxed equation

$$
\lambda_{i} . \nabla_{x} \mathbf{f}_{i}(\varepsilon, x)-\left(\int_{\varepsilon}^{\varepsilon_{\max }} \Sigma(\varepsilon) \mathbf{f}_{i}(\varepsilon, x) d \varepsilon-\sigma_{T}(\varepsilon) \mathbf{f}_{i}(\varepsilon, x)\right)=0
$$

on the interval $\left[\varepsilon^{n+1}, \varepsilon^{n}\right]$, i.e. one computes $\mathbf{f}_{i}^{n+1}$.

3. Finally the influence of the relaxation term is added, which corresponds, when $\tau \rightarrow 0$, to fixing the new value

$$
\Psi^{n+1}=\sum_{i=1}^{J} \mathbf{f}_{i}^{n+1}
$$

One only needs to construct a numerical scheme for (12). Using a simple upwind discretization for the spatial flux and a quadrature for the integral term together with (13) leads to define the following scheme for $\Psi$ 


$$
\frac{\mathbf{F}_{\mathbf{1}_{l+\frac{1}{2}, m}^{n}}^{n}-\mathbf{F}_{\mathbf{1}_{l-\frac{1}{2}, m}^{n}}^{n}}{\Delta x}+\frac{\mathbf{F}_{\mathbf{2}_{l, m+\frac{1}{2}}^{n}}-\mathbf{F}_{\mathbf{2}}^{n}{ }_{l, m-\frac{1}{2}}}{\Delta y}-\left(\sum_{n^{\prime}=1}^{n} \Sigma^{n^{\prime}, n} \Psi_{l, m}^{n^{\prime}} \Delta \varepsilon^{n^{\prime}}-\sigma_{T}^{n} \Psi_{l, m}^{n}\right)=0,
$$

where the fluxes are of HLL ([8]) type

$$
\begin{aligned}
& \mathbf{F}_{\mathbf{1}_{l+\frac{1}{2}, m}^{n}}^{n}=\frac{1}{2}\left[\mathbf{F}_{\mathbf{1}}\left(\Psi_{l+1, m}^{n}\right)+\mathbf{F}_{\mathbf{1}}\left(\Psi_{l, m}^{n}\right)+\left(\Psi_{l+1, m}^{n}-\Psi_{l, m}^{n}\right)\right] \\
& \mathbf{F}_{\mathbf{2}_{l, m+\frac{1}{2}}^{n}}^{n}=\frac{1}{2}\left[\mathbf{F}_{\mathbf{2}}\left(\Psi_{l, m+1}^{n}\right)+\mathbf{F}_{\mathbf{2}}\left(\Psi_{l, m}^{n}\right)+\left(\Psi_{l, m+1}^{n}-\Psi_{l, m}^{n}\right)\right]
\end{aligned}
$$

Recall that the equation (12) is solved backwardly in energy. An iterative solver was proposed in [15] to compute $\Psi_{l, m}^{n}$ at each iteration and a complete analysis of this scheme is postponed to a futur paper.

\section{A numerical experiment}

This test case corresponds to injecting a beam of photons in a 2D medium. The size of the medium is $2 \mathrm{~cm} \times 10 \mathrm{~cm}$, and the beam is $0.5 \mathrm{~cm}$ large and composed of 500 $\mathrm{keV}$ photons. This is modeled by the following kinetic boundary condition

$$
\begin{aligned}
\psi(x, \varepsilon, \Omega) & =10^{10} \exp \left(-\alpha_{\varepsilon}\left(\varepsilon-\varepsilon_{0}\right)^{2}\right) \exp \left(-\alpha_{\mu}\left(\Omega_{1}-1\right)^{2}\right) \mathbf{1}_{B}(x), \text { for } n . \Omega<0, \\
B & =\left\{x=\left(x_{1}, x_{2}\right), \quad x_{1}=0, \quad x_{2} \in[0.75 \mathrm{~cm}, 1.25 \mathrm{~cm}]\right\} .
\end{aligned}
$$

where $\mathbf{1}_{B}$ is the indicator function in the set $B, n$ is the outgoing normal, with $\varepsilon_{0}=$ $500 \mathrm{keV}$, and the constants $\alpha_{\varepsilon}=20000$ and $\alpha_{\mu}=10000$ are chosen arbitrarily large to model a beam. At the discrete level for the moment models, we fix

$$
\begin{aligned}
& \Psi_{0, m}^{n}=10^{10} \exp \left(-\alpha_{\varepsilon}\left(\varepsilon^{n}-\varepsilon_{0}\right)^{2}\right) \int_{S^{2}} \mathbf{m}(\Omega) \exp \left(-\alpha_{\mu}\left(\Omega_{1}-1\right)^{2}\right) d \Omega \mathbf{1}_{B}\left(x_{l, m}\right), \\
& \Psi_{l, 0}^{n}=\Psi_{l_{\max }, m}^{n}=\Psi_{l, m_{\max }}^{n}=0_{\mathbb{R}^{4}} .
\end{aligned}
$$

The density of particles $\rho$ is given by the formula

$$
\rho(x)=\int_{\varepsilon_{\min }}^{\varepsilon_{\max }} \psi^{0}(x, \varepsilon) d \varepsilon .
$$

It is represented on Fig. 1 normalized by the maximum density $\rho_{\max }$, computed using the scheme (14) on the $M_{1}$ equation and compared to a reference Monte Carlo result.

One observes on the Monte Carlo results on Fig. 1 that the photons travel through the medium almost in straightline. They are only rarely scattered and the beam remains sharp deep in the medium. However, the $M_{1}$ results with the scheme (14) are 


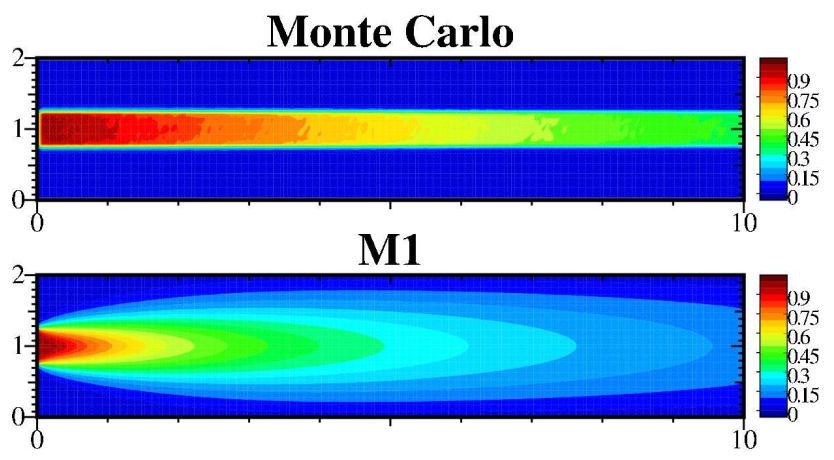

Fig. 1 Density $\rho$ obtained with the Monte Carlo solver (top) and the $M_{1}$ model (below).

significantly different. One may observe a beam shape, however this beam is very diffused especially in the direction transverse to the beam. This effect is actually a numerical artefact due to the relaxation parameters chosen (11) and the next section is devoted to correcting it.

\section{A correction to accurately model transverse diffusion}

In practice, the relaxation method can be used under the stability condition (10) on the relaxation speeds. However, the relaxation method is known to be overdiffusive when the relaxation speeds $\lambda_{j}$ are too large. In practice, the larger is the $\lambda_{i}$ the more stable is the resulting scheme, but the more diffusive it is. This can be verified by reproducing the computations of $[14,1]$. The present correction uses similar ideas as the ones proposed in $[6,2]$.

\subsection{Bounds on the eigenvalues of the Jacobian of the flux}

The relaxation speeds were chosen to be of norm $\left|\lambda_{j}\right|=N$ which was enough to satisfy (10) according to Proposition 2. However, those bounds are too large when $\Psi$ is the moment vector of a beam. Consider a beam of direction $e_{1}$ modeled by

$$
\Psi=\int_{S^{2}} \mathbf{m}(\Omega) \exp \left(-\alpha_{\mu}\left(\Omega_{1}-1\right)\right) d \Omega .
$$

The spectral radius of the Jacobian of the flux $\mathbf{F}_{\mathbf{2}}$ transverse to the direction of the beam is zero. Indeed, using the even and odd character of the following functions, one finds that for all $V \in \mathbb{R}^{4}$ 


$$
\begin{aligned}
V^{T} \partial_{\Lambda} \mathbf{F}_{\mathbf{2}}(\Psi) V & =\int_{S^{2}} \Omega_{2}(V \cdot \mathbf{m}(\Omega))^{2} \exp \left(-\alpha_{2}\left(\Omega_{1}-1\right)\right) d \Omega=0, \\
V^{T} \partial_{\Lambda} \Psi V & =\int_{S^{2}}(V \cdot \mathbf{m}(\Omega))^{2} \exp \left(-\alpha_{\mu}\left(\Omega_{1}-1\right)\right) d \Omega>0 .
\end{aligned}
$$

This means that the eigenvalues of $\partial_{\Lambda} \mathbf{F}_{2}(\Psi)$ are all zeros while $\partial_{\Lambda} \Psi$ is strictly positive. Therefore the eigenvalues of the Jacobian $\partial_{\Psi} \mathbf{F}_{2}=\partial_{\Lambda} \mathbf{F}_{2}(\Psi)\left(\partial_{\Lambda} \Psi\right)^{-1}$ of the transverse flux are all zero.

Those eigenvalues can actually be computed analytically (as the Jacobian of the flux is a $4 \times 4$ matrix, those are the roots of a quartic). For the present numerical purpose, we only compute bounds on those eigenvalues that are easily computable and implementable. For writing consideration, those computations are gathered in Appendix. In the rest of this paper, the minimum and maximum of $\operatorname{Sp}\left(\partial_{\Psi} \mathbf{F}_{\mathbf{n}}\right)$ are called $b_{-}$and $b_{+}$and are given functions of the direction $n$ of the flux $\mathbf{F}_{\mathbf{n}}$ and of the normalized first order moment

$$
N^{1}=\frac{\psi^{1}}{\psi^{0}}
$$

\subsection{The modified relaxation parameters}

Based on those bounds, we propose to modify the relaxation parameters (11) into

$$
\begin{aligned}
& \lambda_{1}=\left(b_{1}, 0\right), \quad \lambda_{2}=\left(b_{2}, 0\right), \quad \lambda_{3}=\left(0, b_{3}\right), \quad \lambda_{4}=\left(0, b_{4}\right), \\
& \mathbf{M}_{\mathbf{1}}=\frac{\left|b_{1}\right|}{\left|b_{1}\right|+\left|b_{2}\right|}\left(\frac{\Psi}{2}+\frac{\mathbf{F}_{\mathbf{1}}}{\left|b_{1}\right|}\right), \quad \mathbf{M}_{\mathbf{2}}=\frac{\left|b_{2}\right|}{\left|b_{1}\right|+\left|b_{2}\right|}\left(\frac{\Psi}{2}+\frac{\mathbf{F}_{\mathbf{1}}}{\left|b_{2}\right|}\right), \\
& \mathbf{M}_{\mathbf{3}}=\frac{\left|b_{3}\right|}{\left|b_{3}\right|+\left|b_{4}\right|}\left(\frac{\Psi}{2}+\frac{\mathbf{F}_{\mathbf{2}}}{\left|b_{3}\right|}\right), \quad \mathbf{M}_{\mathbf{4}}=\frac{\left|b_{4}\right|}{\left|b_{3}\right|+\left|b_{4}\right|}\left(\frac{\Psi}{2}+\frac{\mathbf{F}_{\mathbf{2}}}{\left|b_{4}\right|}\right) .
\end{aligned}
$$

The coefficients $b_{i}$ can still be chosen such that the parameters (15) satisfy (10) and such that they are smaller than those in (11).

Recall that we also required the Maxwellians $\mathbf{M}_{\mathbf{i}} \in \mathscr{R}_{\mathbf{m}}$ to be realizable. In practice, this leads to an additional requirement on the bounds $b_{1}, b_{2}, b_{3}$ and $b_{4}$. For the $M_{1}$ model, those requirements can easily be computed using (7), for $\mathbf{M}_{\mathbf{1}}$ it reads

$$
\left(\frac{1}{2}+\frac{N_{1}^{1}}{\left|b_{1}\right|}\right)^{2}>\left|\frac{1}{2} N^{1}+\frac{N^{2} \cdot e_{1}}{\left|b_{1}\right|}\right|^{2} .
$$

Solving this quadratic inequality leads to chose $b_{1}$ such that

$$
\begin{aligned}
& \left|b_{1}\right|>\max \left(0, b_{\min }\left(N^{1}, e_{1}\right)\right), \quad b_{\min }\left(N^{1}, n\right):=\frac{-\beta_{n}-\sqrt{\beta_{n}^{2}-\alpha \gamma_{n}}}{\alpha} \\
& \alpha=\frac{1-\left|N^{1}\right|^{2}}{4}, \quad \beta_{n}=\frac{1}{2}\left(N^{1} . n-N^{1} .\left(N^{2} n\right)\right), \quad \gamma_{n}=\left(N^{1} . n\right)^{2}-\left|N^{2} n\right|^{2} .
\end{aligned}
$$


Similar computations hold for $b_{2}, b_{3}$ and $b_{4}$ which lead to fixing the bounds

$$
\begin{aligned}
& b_{1}(\Psi)=\max \left(10^{-8}, \quad b_{+}\left(N^{1}, e_{1}\right), \quad b_{\min }\left(N^{1}, e_{1}\right)\right), \\
& b_{2}(\Psi)=\min \left(-10^{-8}, \quad b_{-}\left(N^{1}, e_{1}\right), \quad-b_{\min }\left(N^{1},-e_{1}\right)\right), \\
& b_{3}(\Psi)=\max \left(10^{-8}, \quad b_{+}\left(N^{1}, e_{2}\right), \quad b_{\min }\left(N^{1}, e_{2}\right)\right), \\
& b_{4}(\Psi)=\min \left(-10^{-8}, \quad b_{-}\left(N^{1}, e_{2}\right), \quad-b_{\min }\left(N^{1},-e_{2}\right)\right),
\end{aligned}
$$

where the constants $\pm 10^{-8}$ are chosen arbitrarily low to avoid divisions by zero and $e_{1}$ and $e_{2}$ are the Cartesian axes.

\subsection{The new numerical scheme}

Using the relaxation parameters (15) to construct a scheme for (3) leads to rewrite the fluxes of the form ([6])

$$
\begin{aligned}
& \mathbf{F}_{l+\frac{1}{2}, m}^{n+1}=\frac{1}{\left|b_{1, l+\frac{1}{2}, m}^{n+1}\right|+\left|b_{2, l+\frac{1}{2}, m}^{n+1}\right|}\left[\left|b_{2, l+\frac{1}{2}, m}^{n+1}\right| \mathbf{F}_{\mathbf{1}}\left(\Psi_{l+1, m}^{n+1}\right)+\left|b_{1, l+\frac{1}{2}, m}^{n+1}\right| \mathbf{F}_{\mathbf{1}}\left(\Psi_{l, m}^{n+1}\right)\right. \\
& \left.+\left|b_{1, l+\frac{1}{2}, m}^{n+1} b_{2, l+\frac{1}{2}, m}^{n+1}\right|\left(\Psi_{l+1, m}^{n+1}-\Psi_{l, m}^{n+1}\right)\right], \\
& \mathbf{F}_{l, m+\frac{1}{2}}^{n+1}=\frac{1}{\left|b_{3, l, m+\frac{1}{2}}^{n+1}\right|+\left|b_{4, l, m+\frac{1}{2}}^{n+1}\right|}\left[\left|b_{4, l, m+\frac{1}{2}}^{n+1}\right| \mathbf{F}_{\mathbf{2}}\left(\Psi_{l, m+1}^{n+1}\right)+\left|b_{3, l, m+\frac{1}{2}}^{n+1}\right| \mathbf{F}_{\mathbf{2}}\left(\Psi_{l, m}^{n+1}\right)\right. \\
& \left.+\left|b_{3, l, m+\frac{1}{2}}^{n+1} b_{4, l, m+\frac{1}{2}}^{n+1}\right|\left(\Psi_{l, m+1}^{n+1}-\Psi_{l, m}^{n+1}\right)\right] \text {, } \\
& b_{1, l+\frac{1}{2}, m}^{n+1}=\max \left(b_{1}\left(\Psi_{l, m}^{n+1}\right), b_{1}\left(\Psi_{l+1, m}^{n+1}\right)\right), \quad b_{2, l+\frac{1}{2}, m}^{n+1}=\min \left(b_{2}\left(\Psi_{l, m}^{n+1}\right), b_{2}\left(\Psi_{l+1, m}^{n+1}\right)\right), \\
& b_{3, l, m+\frac{1}{2}}^{n+1}=\max \left(b_{3}\left(\Psi_{l, m}^{n+1}\right), b_{3}\left(\Psi_{l+1, m}^{n+1}\right)\right), \quad b_{4, l, m+\frac{1}{2}}^{n+1}=\min \left(b_{4}\left(\Psi_{l, m}^{n+1}\right), b_{4}\left(\Psi_{l+1, m}^{n+1}\right)\right)
\end{aligned}
$$

in the scheme (14). The numerical fluxes are now defined locally as a function of the unknowns and the fluxes which allows to better capture the diffusion effects. One may verify that the coefficients $\left|b_{1, l+\frac{1}{2}, m}^{n+1} b_{2, l+\frac{1}{2}, m}^{n+1}\right|$ before the terms $\left(\Psi_{l+1, m}^{n+1}-\Psi_{l, m}^{n+1}\right)$, responsible for the numerical diffusion, in the definition of the numerical fluxes (16) are lower than the one in the scheme (14). 


\subsection{Results with the modified scheme}

Using this modified scheme on the test case of Section 4 provides the dose result on Fig. 2 with the computational times in Table 1.

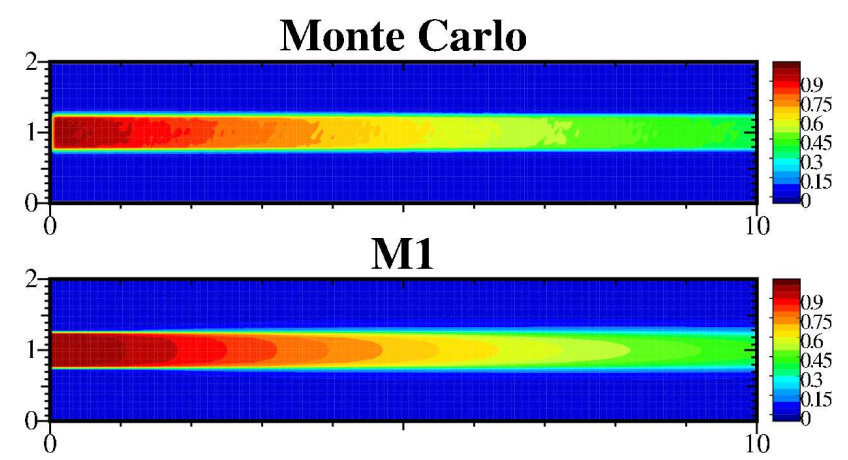

Fig. 2 Density $\rho$ obtained with the Monte Carlo solver (top) and the $M_{1}$ model (below) with the modified relaxation parameters.

\begin{tabular}{|c||c|c|c|}
\hline Solver & Monte Carlo & $M_{1}$ solver & modified $M_{1}$ solver \\
\hline \hline Computation times & 14 hours & $49.78699 \mathrm{sec}$ & $204.1239 \mathrm{sec}$ \\
\hline
\end{tabular}

Table 1 Computational times with the different numerical methods.

The results with the modified relaxation parameters are much closer to the reference Monte Carlo results. The diffusion in the tranverse direction is much lower than the one on Fig. 1 and the beam stays sharp through the medium.

The computational cost is considerably lower with the present numerical method compared to the reference Monte Carlo code. The computational time is although higher when using the new relaxation parameters (15) than when using the one in (11). This is actually due to the method used to compute $\Psi_{l, m}^{n}$ from (14) or (16). The conditioning of problem (14) is simply better than the one of (16) which explains the difference of computational times.

\section{Conclusion}

A numerical method for the transport of photons in a human-sized medium was proposed. This method aims to solve $M_{1}$ equations. When modelling a beam of photons in such a medium, the main difficulties of those simulations is due to the fact that the diffusion phenomena in the direction of the beam and the ones normal to the beam have considerably different magnitude. Standard methods present a numerical diffusion which is considerably overestimated in the tranverse direction. This effect affects the accuracy of the simulation. First a numerical scheme was proposed 
for solving the transport equation, then it was improved to accurately capture the diffusion effect in the transverse direction.

Acknowledgements The author would like to acknowledge K. Küpper and G. Birindelli for performing the Monte Carlo simulations used as reference results in this paper.

\section{Appendix : Computation of bounds on the eigenvalues of the Jacobian of the flux}

Using rotational invariance and a normalization (see [11] for details), this closure can also be rewritten under the form

$$
\psi^{2}=\psi^{0}\left[\frac{1-\chi}{2} I d+\frac{3 \chi-1}{2} \frac{\psi^{1} \otimes \psi^{1}}{\left|\psi^{1}\right|^{2}}\right]
$$

where the Eddington factor $\chi$ is a scalar function of the scalar $\left|\psi^{1}\right| / \psi^{0}$.

Consider that $\psi^{1}$ is colinear to $e_{1}$ (otherwise just use a rotation to work in such a reference frame). Using the form (17) of the closure, the fluxes in the direction $e_{1}$ and in the transverse direction $e_{2}$ read

$$
\Psi=\left(\psi^{0}, \psi^{1}\right), \quad \mathbf{F}_{\mathbf{n}}(\Psi)=\left(\psi^{1} . n, \frac{\psi^{0}}{2}\left[(1-\chi) n+(3 \chi-1) \frac{\left(\psi^{1} \cdot n\right) \psi^{1}}{\left|\psi^{1}\right|^{2}}\right]\right)
$$

Chose a reference frame such that $\psi^{1}=\psi_{1}^{1} e_{1}$ with $\psi_{1}^{1} \geq 0$. In this reference frame, the spectrum of the Jacobian of the flux $\mathbf{F}_{\mathbf{1}}$ along the direction $e_{1}$ (direction of the beam) and along $e_{2}$ (direction normal to the beam) read

$$
\begin{aligned}
& S p\left(\partial_{\Psi}\left(\mathbf{F}_{\mathbf{1}}(\Psi)\right)\right)=\left(\frac{3 \chi-1}{2 N_{1}^{1}}, \frac{\chi^{\prime} \pm \sqrt{\chi^{\prime 2}+4\left(\chi-N_{1}^{1} \chi^{\prime}\right)}}{2}\right) \\
& S p\left(\partial_{\Psi}\left(\mathbf{F}_{2}(\Psi)\right)\right)=\left(0, \pm \sqrt{\frac{1-\chi+N_{1}^{1} \chi^{\prime}-\frac{3 \chi-1}{2 N_{1}^{1}} \chi^{\prime}}{2}}\right) .
\end{aligned}
$$

Now, in order to come back to the computations in any reference frame, one can simply use a rotation $R$ such that $R \psi^{1}=\psi_{1}^{1} e_{1}$. One has

$$
\begin{aligned}
\partial_{\Psi}\left(\mathbf{F}_{\mathbf{n}}(\Psi)\right) & =\partial_{\left(\psi^{0}, R \psi_{1}^{1} e_{1}\right)}\left(\mathbf{F}_{\mathbf{n}}\left(\psi^{0}, R \psi_{1}^{1} e_{1}\right)\right) \\
& =R_{2} \partial_{\Psi} \mathbf{F}_{\mathbf{R}^{\mathbf{T}}}\left(\psi^{0},\left|\psi^{1}\right| e_{1}\right) R_{2}^{T}, \quad R_{2}=\left(\begin{array}{cc}
1 & 0_{\mathbb{R}^{3}} \\
0_{\mathbb{R}^{3}} & R
\end{array}\right) .
\end{aligned}
$$

The spectrum of such a matrix can be bounded using the previous computations 


$$
\begin{aligned}
\operatorname{Sp}\left(\partial_{\Psi}\left(\mathbf{F}_{\mathbf{n}}(\Psi)\right)\right) & \subset\left[b_{-}, b_{+}\right] \\
b_{-}\left(N^{1}, n\right) & =(1-\theta) \min S_{t}\left(\left|N^{1}\right|\right)+\theta \min S_{n}\left(\left|N^{1}\right|\right), \\
b_{+}\left(N^{1}, n\right) & =(1-\theta) \max S_{t}\left(\left|N^{1}\right|\right)+\theta \max S_{n}\left(\left|N^{1}\right|\right), \quad \theta=\frac{N^{1} . n}{\left|N^{1}\right|}
\end{aligned}
$$

The exact bounds $b_{-}$and $b_{+}$of $\operatorname{Sp}\left(\partial_{\Psi} \mathbf{F}_{\mathbf{n}}(\Psi)\right)$ could be computed analytically as the eigenvalues of the $4 \times 4$ matrix $\partial_{\Psi}\left(\mathbf{F}_{\mathbf{n}}(\Psi)\right)$. However using such analytical formulae may introduce errors at the numerical level that may be non-negligible. Computing the bounds in (18) is easier, and they are sufficient for the present applications.

\section{References}

1. D. Aregba-Driollet and R. Natalini. Discrete kinetic schemes for multidimensional systems of conservation laws. SIAM J. Numer. Anal., 6:1973-2004, 2000.

2. C. Berthon, P. Charrier, and B. Dubroca. An HLLC scheme to solve the $M_{1}$ model of radiative transfer in two space dimensions. J. Sci. Comput., 31(3):347-389, 2007.

3. J. Borwein and A. Lewis. Duality relationships for entropy-like minimization problems. SIAM J. Control Optim., 29(2):325-338, 1991.

4. C. M. Davisson and R. D. Evans. Gamma-ray absorption coefficients. Rev. Mod. Phys., 1952.

5. R. Duclous, B. Dubroca, and M. Frank. A deterministic partial differential equation model for dose calculation in electron radiotherapy. Phys. Med. Biol., 55:3843-3857, 2010.

6. B. Einfeldt. On godunov-type methods for gas dynamics. SIAM J. Numer. Anal., 25(2):294318,1988

7. K. O. Friedrichs and P. D. Lax. Systems of conservation equations with a convex extension. Proc. Nat. Acad. Sci., 68(8):1686-1688, 1971.

8. A. Harten, P. Lax, and B. Van Leer. On upstream differencing and Gudonov-type schemes for hyperbolic conservation laws. SIAM Rev., 25(1):35-61, 1983.

9. M. Junk. Maximum entropy for reduced moment problems. Math. Mod. Meth. Appl. S., 10(1001-1028):2000, 1998.

10. D. Kershaw. Flux limiting nature's own way. Technical report, Lawrence Livermore Laboratory, 1976.

11. C. D. Levermore. Relating Eddington factors to flux limiters. J. Quant. Spectros. Radiat. Transfer, 31:149-160, 1984.

12. C. D. Levermore. Moment closure hierarchies for kinetic theories. J. Stat. Phys., 83(56):1021-1065, 1996.

13. G. N. Minerbo. Maximum entropy Eddington factors. J. Quant. Spectros. Radiat. Transfer, 20:541-545, 1978.

14. R. Natalini. A discrete kinetic approximation of entropy solutions to multidimensional scalar conservation laws. J. Differ. Equations, 148(2):292 - 317, 1998.

15. T. Pichard, G.W. Alldredge, S. Brull, B. Dubroca, and M. Frank. An approximation of the $M_{2}$ closure: Application to radiotherapy dose simulation. J. Sci. Comput., to appear.

16. T. Pichard, D. Aregba-Driollet, S. Brull, B. Dubroca, and M. Frank. Relaxation schemes for the $M_{1}$ model with space-dependent flux: application to radiotherapy dose calculation. Commun. Comput. Phys., 19:168-191, 2016.

17. J. Schneider. Entropic approximation in kinetic theory. ESAIM-Math. Model. Num., 38(3):541-561, 2004.

18. C. P. South, M. Partridge, and P. M. Evans. A theoretical framework for prescribing radiotherapy dose distributions using patient-specific biological information. Med. Phys., 35(10):45994611, 2008. 